\title{
Circling in on Convective Organization
}

Härter, Jan Olaf Mirko; Böing, Steven J.; Henneberg, Olga Cassandra; Nissen, Silas Boye

Published in:

Geophysical Research Letters

DOI:

10.1029/2019GL082092

Publication date:

2019

Document version

Publisher's PDF, also known as Version of record

Citation for published version (APA):

Härter, J. O. M., Böing, S. J., Henneberg, O. C., \& Nissen, S. B. (2019). Circling in on Convective Organization.

Geophysical Research Letters, 46, 1-11. https://doi.org/10.1029/2019GL082092 


\section{Geophysical Research Letters}

\author{
RESEARCH LETTER \\ 10.1029/2019GL082092 \\ Key Points: \\ - Convection is often initiated by the \\ collision of three cold pools \\ - A model based on expanding and \\ colliding circles captures \\ the convective scale increase \\ - This simple model can produce \\ clustering of precipitation cells
}

Supporting Information:

- Supporting Information S1

Correspondence to:

J. O. Haerter and S. B. Nissen,

haerter@nbi.ku.dk;

silas@nbi.ku.dk

Citation:

Haerter, J. O., Böing, S. J.,

Henneberg, O., \& Nissen, S. B. (2019). Circling in on convective organization. Geophysical Research Letters, 46, 7024-7034. https://doi.org/10.1029/ 2019GL082092

Received 16 JAN 2019 Accepted 16 MAY 2019 Accepted article online 23 MAY 2019 Published online 21 JUN 2019

\section{Circling in on Convective Organization}

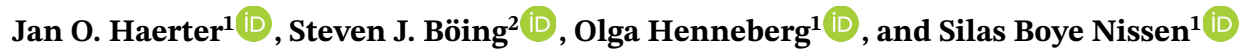 \\ ${ }^{1}$ Niels Bohr Institute, University of Copenhagen, Copenhagen, Denmark, ${ }^{2}$ School of Earth and Environment, \\ University of Leeds, Leeds, UK
}

\begin{abstract}
Cold pools (CPs) contribute to convective organization. However, it is unclear by which mechanisms organization occurs. By using a particle method to track CP gust fronts in large eddy simulations, we characterize the basic collision modes between CPs. Our results show that CP interactions, where three expanding gust fronts force an updraft, are key at triggering new convection. Using this, we conceptualize CP dynamics into a parameter-free mathematical model: circles expand from initially random points in space. Where two expanding circles collide, a stationary front is formed. However, where three expanding circles enclose a single point, a new expanding circle is seeded. This simple model supports three fundamental features of CP dynamics: precipitation cells constitute a spatially interacting system, CPs come in generations, and scales steadily increase throughout the diurnal cycle. Finally, this model provides a framework for how CPs act to cause convective self-organization, clustering, and extremes.
\end{abstract}

Plain Language Summary Cold pool (CP) dynamics constitutes a crucial organizing mechanism for midlatitude and tropical clouds - they play a key role in the lead-up to extreme events and may influence how such events behave in a changing climate. CPs are dense air masses that form under precipitating thunderstorm clouds. Under gravity, CPs spread along the surface and stimulate new precipitation events when they collide with other CPs. We show that CP interaction can be captured by a simple model, where circles grow in space and form new circles when three of them collide. Generalizing to thousands of initial circle centers, the dynamics of these circles gives a steady scale increase over time, similar to the one found in high-resolution atmospheric simulations. In summary, we introduce a cloud-organizing mechanism that forms the basis for extreme convective precipitation events, such as those implicated in flash floods.

\section{Introduction}

The transition from a nonprecipitating to a precipitating atmosphere fundamentally alters the organization of its cloud field (Feingold et al., 2010; Haerter et al., 2017; Koren \& Feingold, 2011; Moseley et al., 2016; Tompkins, 2001; Zuidema et al., 2017). Cold pools (CPs) are produced when rain evaporates or other hydrometeors melt, forming boundary layer air that is denser than the surrounding air. Due to its larger density and gravity CP air accelerates toward the surface and is then forced to spread laterally. From large eddy simulations (LES) and from observations it is well established that such CP outflows act to organize the subcloud temperature, moisture, and wind fields (de Szoeke et al., 2017; Droegemeier \& Wilhelmson, 1985; Feng et al., 2015; Khairoutdinov \& Randall, 2006; Purdom, 1982; Schlemmer \& Hohenegger, 2015; Tompkins, 2001; Torri \& Kuang, 2016). Apart from influencing the thermodynamic stability in the boundary layer (Tompkins, 2001; Torri et al., 2015), by which CP edges may become relatively buoyant, CPs play a crucial role as a dynamic trigger for convection (Haerter \& Schlemmer, 2018; Jeevanjee \& Romps, 2015; Moseley et al., 2016; Torri et al., 2015). Moreover, studies have shown that in particular the bands of collisions between CPs are collocated with strong updrafts (Böing et al., 2012; Feng et al., 2015; Lima \& Wilson, 2008). CP fronts caused by deep convection can travel at velocities on the order of $10 \mathrm{~m} / \mathrm{s}$ radially in tropical environments (CPs can move faster for midlatitude storms; Drager \& van den Heever, 2017; Romps \& Jeevanjee, 2016; Zuidema et al., 2017). When CPs collide, momentum conservation can displace moist air vertically (Cafaro \& Rooney, 2018; Moseley et al., 2016; Torri et al., 2015). This air thereby can condense, overcome the level of free convection, and rise further due to increased buoyancy.
(C)2019. American Geophysical Union. All Rights Reserved. 
To better represent the effects of CPs on convection and obtain a more realistic timing of the diurnal cycle of precipitation, some general circulation models include a statistical representation of CP processes, for example, by describing the population of CPs as circular objects of identical radii (Grandpeix \& Lafore, 2010; Grandpeix et al., 2010; Rio et al., 2009). Furthermore, extreme convective precipitation increases at rates beyond that of the saturation mixing ratio, as expressed through the Clausius-Clapeyron relation (Berg et al., 2013; Guerreiro et al., 2018; Haerter \& Schlemmer, 2018; Lenderink \& van Meijgaard, 2008). Explanations may well require arguments involving CP-induced self-organization (Haerter \& Schlemmer, 2018; Moseley et al., 2016).

CP dynamics has been described by simplified models (Bengtsson et al., 2013; Böing, 2016), showing that they can act as two-dimensional cellular automata. However, the self-organization of convection is still poorly understood, especially how small-scale interaction may give rise to large-scale organization (Moncrieff, 2010). In previous simulations of the diurnal cycle of convection (Haerter et al., 2017), it was found using a spatial correlation function that the velocity field is initially characterized by Rayleigh-Bénard-type convection during the morning hours, with lateral scales set by the boundary layer height (Mellado, 2012). As soon as precipitation sets in near midday, this correlation function shows a systematic increase of the distance between updrafts, indicating that the onset of precipitation leads to an increase of typical scales. Comparing various simulations suggested that the scale increases approximately linearly with time, and the rate of increase is not strongly dependent on the simulation boundary conditions.

Our aim is to isolate the key interactions between CPs from LES to incorporate these in a simple conceptual model, and to analyze how they influence the cloud field.

\section{Data and Methods}

\subsection{LES Data}

We use simulation data from the University of California, Los Angeles, LES code (UCLA-LES; Stevens et al., 2005) for the diurnal cycle of convection under spatially homogeneous surface boundary conditions and a prescribed, temporally varying surface temperature cycle. The model domain size is $192 \mathrm{~km} \times 192 \mathrm{~km}$ horizontally and $17 \mathrm{~km}$ vertically. The simulation is run at $200-\mathrm{m}$ horizontal grid spacing and has 75 vertical model levels stretching from $100 \mathrm{~m}$ at the surface to $400 \mathrm{~m}$ at the domain top. The model uses a two-moment microphysics scheme (Seifert \& Beheng, 2006) and a delta four-stream radiation code (Pincus \& Stevens, 2009). Surface latent and sensible heat fluxes are modeled using Monin-Obukhov similarity theory. The model time step ranges from 1 to $5 \mathrm{~s}$. The atmosphere is initialized by vertical profiles for temperature and humidity observed at Lindenberg, Germany, during the summers of 2007 and 2008 (Figure S3 of Haerter $\&$ Schlemmer, 2018). No ambient wind or pressure gradients are applied. For the results in Figure 1 and supporting information Figure S4, the surface temperature $T_{s}(t)$ varies sinusoidally with an amplitude of $10 \mathrm{~K}$, a period of $24 \mathrm{hr}$, a peak at $12 \mathrm{hr}$ (noon), and a mean $\bar{T}_{s}=27^{\circ} \mathrm{C}$. The comparison of scale increases in Figure 2a makes use of data from six LES with distinct boundary conditions. The symbols in Figure 2a refer to $\bar{T}_{s}=23^{\circ} \mathrm{C}(\mathrm{CTR}), 24^{\circ} \mathrm{C}(\mathrm{P} 1 \mathrm{~K}), 25^{\circ} \mathrm{C}(\mathrm{P} 2 \mathrm{~K})$, and $27{ }^{\circ} \mathrm{C}(\mathrm{P} 4 \mathrm{~K}$, identical to the one discussed above); as well as a simulation where the period of forcing was increased to $48 \mathrm{hr}$ (LD) but otherwise maintaining CTR; and finally a less stable initial atmospheric lapse rate (LAPSE). These simulations and the data plotted in Figure 2a are described in more detail in Haerter et al. (2017) and Moseley et al. (2016).

\subsection{Particle Method for CP Tracking}

We here employ a method for tracking of CPs, which capitalizes on the observation that forced uplift is crucial in creating the sharp updrafts required for new cell initiation. Our method is described as follows.

\section{Defining Rain Events}

A rain cell is defined as a spatially contiguous patch formed by grid boxes which each exceed an accumulated precipitation intensity limit $I_{0}$ (set to $I_{0}=0.5 \mathrm{~mm} / \mathrm{hr}$ ) during an interval $\Delta t$, with $\Delta t=5 \mathrm{~min}$. A lower limit of 10 grid boxes is required for a patch to be considered in the analysis. Reasonable intensity and area limits have recently been discussed (Moseley et al., 2019). All rain cells are tracked using the Iterative Rain Cell Tracking method (Moseley et al., 2013). Rain tracks are formed by identifying rain cells that spatially overlap from one time step to the next. Each rain track receives a unique identifier, called the track ID (details: Moseley et al., 2013). Merging and splitting events are handled through a unit threshold ratio, by which the largest cell is continued by maintaining its ID (details: Moseley et al., 2019). 

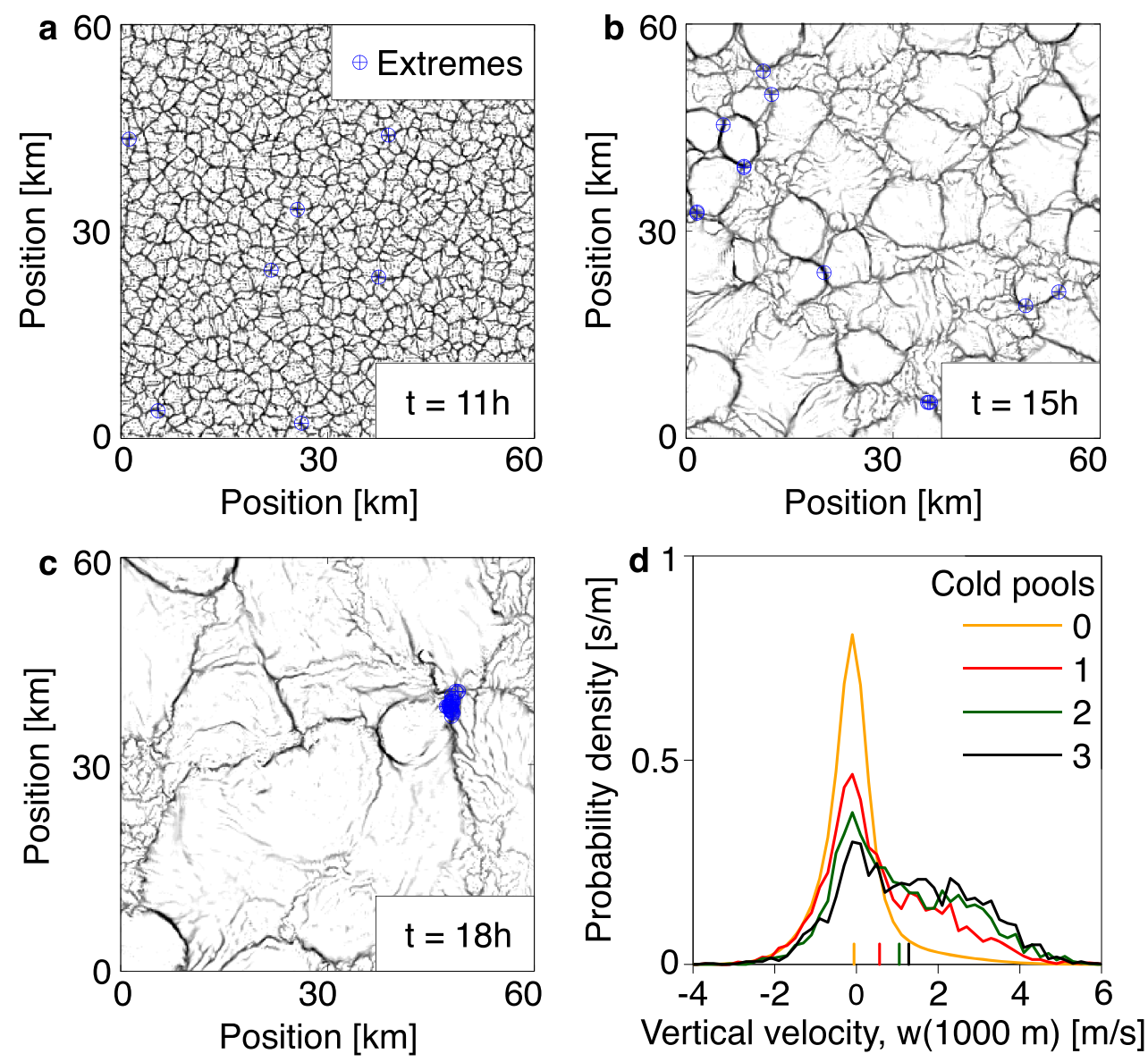

Figure 1. Analysis of cold pool collisions. (a-c) Horizontal cross sections of large-eddy simulated near-surface vertical velocity $w(100 \mathrm{~m})$. Darker shades qualitatively highlight larger vertical velocity (quantification: see Figure S5). Blue symbols denote locations with $w(1,000 \mathrm{~m})$ exceeding the 99.98th percentile. Panels correspond to times near precipitation onset $(11 \mathrm{hr})$, the time near greatest domain mean intensity $(15 \mathrm{hr})$, and for decaying mean intensity $(18 \mathrm{hr}$ ), respectively. (details: Figure S3). Note that the axis scales are identical in all three panels, highlighting a progressive scale increase. (d) Probability density functions conditional on the geometric location within cold pools; the legend labels the number of cold pools contributing, for all times where precipitation was present in the large eddy simulations (all $t$ where $I(t)>0$ in Figure S3). Short vertical lines near the horizontal axis show means of corresponding probability density functions. (Technical details: section 2 ).

\section{Tracking CP Gust Fronts and Identifying Collisions}

We emit particles within the lowest model level at the boundary pixels of the identified rain cells and advect these particles radially with the horizontal flow. The particles are associated with the respective rain event by assigning them a matching track ID. (details: section S2).

By collocating particles corresponding to different CPs, collisions between CPs can be mapped out systematically (compare Figure S4c). The collocation is done by scanning a window of $n_{g} \times n_{g}$ grid boxes for the simultaneous occurrence of tracers belonging to distinct CPs (compare Figure S4); in Figure 1d the strictest and simplest case is presented $\left(n_{g}=1\right)$, that is, using only a single grid box for each tracer. Examples for $n_{g}=3$ are shown in Figure S6. In cases where more than a threshold number of particles (set to 10 in Figure 1d) are identified, the diversity of CP indices within the window is quantified-leading to the classifications into classes of zero, one, two, or three CPs (Figure 1). The sensitivity of our results to $n_{g}$, the threshold number of particles, and the time of the model day were investigated, showing robustness regarding the ordering of the interaction modes (Figure S6). In particular, as the day progresses, the distribution function corresponding to $2 \mathrm{CP}$ collisions becomes more and more similar to that of $3 \mathrm{CP}$ collisions (Figure S6f and S6i). 

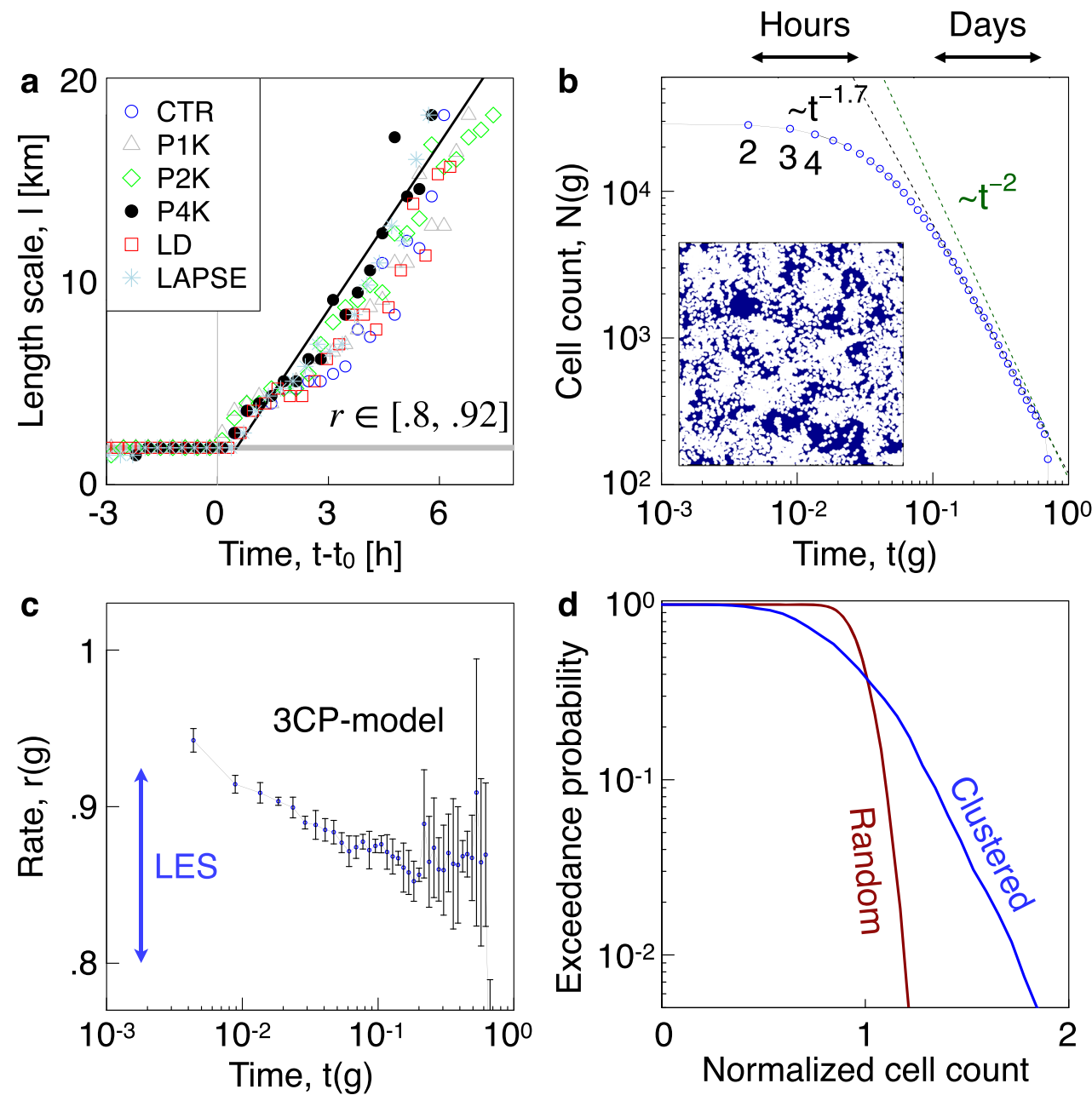

Figure 2. Cold pool dynamics in the conceptual model. (a) Increase of spatial scales for large eddy simulations (LES) for various boundary conditions (details: section 2.1). The $t_{0}$ marks the time of precipitation onset for each simulation. The speed of scale increase $v \equiv c_{0}(1-r)$ is the slope of a fit to the data points (exemplified for P4K, black line, where $v \approx 0.8 \mathrm{~m} / \mathrm{s}$; Haerter et al., 2017). Using $v=0.8 \mathrm{~m} / \mathrm{s}$, equation (4), and $c_{0} \in[4,10] \mathrm{m} / \mathrm{s}$, estimated from LES, the range, blue arrow in (c), is obtained. Plot modified from Haerter et al. (2017). (b) Number of cells per generation $N(g)$ plotted against the corresponding average creation time $t(g) \equiv N(g)^{-1} \sum_{i} t_{i}^{(g)}$ : each point represents the number of cells in a given generation. Time is measured in units of $L / c_{0}$; arrows along panel top show approximate time scales for typical values of $c_{0}$ and initial cell density. The black and green straight dashed lines represent power laws with exponents -1.7 and -2 , respectively. Labels along the curve indicate several generation numbers for clarity. Inset: conceptual model results in a $800 \times 800 \mathrm{~km}^{2}$ subarea after approximately $3 \mathrm{hr}$ from initial random seeding of cells, during a 3-hr interval. We assume an initial average cell-to-cell distance of $10 \mathrm{~km}$ and $c_{0} \approx 10 \mathrm{~m} / \mathrm{s}$. (details: section S3).

(c) Replication rate $r(g$ ) from 3CP model (black curve) and estimate from LES (blue arrow), using (a). (d) Cumulative distribution functions for simulated (blue) and randomized (red) cell count for data as in the inset to (b). Cell count is normalized to the mean cell count in the subdomain (details: section 3). Note the logarithmic vertical axes in (b) and (d) and the logarithmic horizontal axes in (b) and (c).

\section{Conceptual Model}

Our conceptual model represents $\mathrm{CP}$ gust fronts as expanding circles, where the origin of each circle is a given rain event. At locations where circles meet, new rain events can form, which then cause new circles to emerge.

Model dynamics. More specifically, $N$ initial cells, referred to as generation one, are seeded at random locations in a two-dimensional square of area $A \equiv L \times L$, where $L$ is the domain length. We impose cyclic boundary conditions in both horizontal directions. The cells grow as circles, representing CP gust fronts. All circle radii are initialized as zero and increase linearly in time at constant speed $c_{0}$. Our model assumes 
a

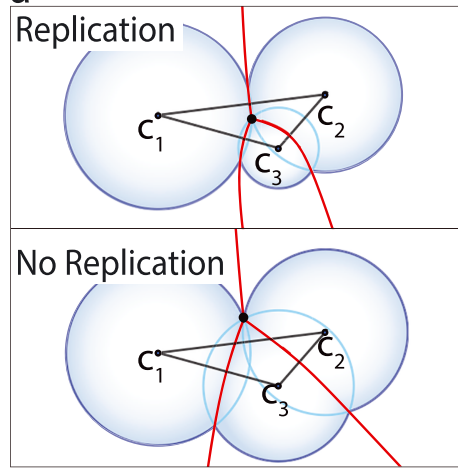

b

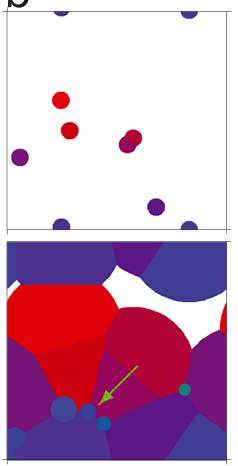

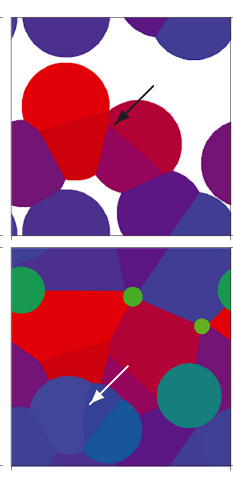

$\stackrel{8}{\circ}$

○

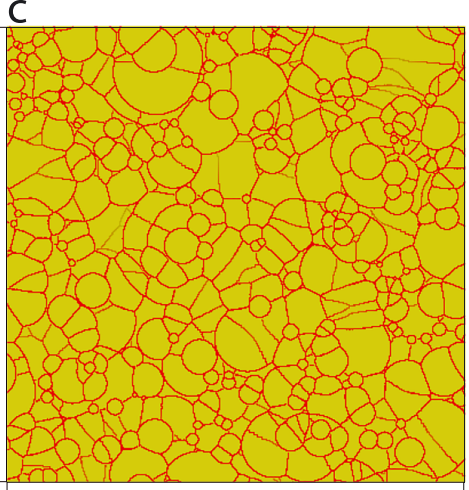

$8 \mathrm{~d}$

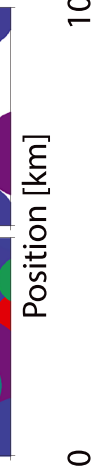

0 0

4 7

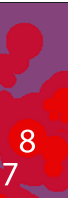

$5 \longdiv { 6 }$

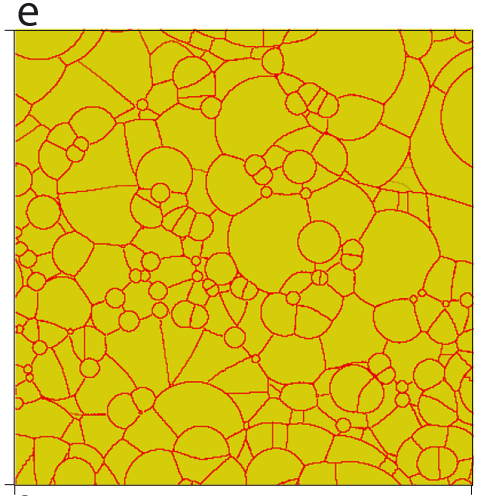

f 11 12 13
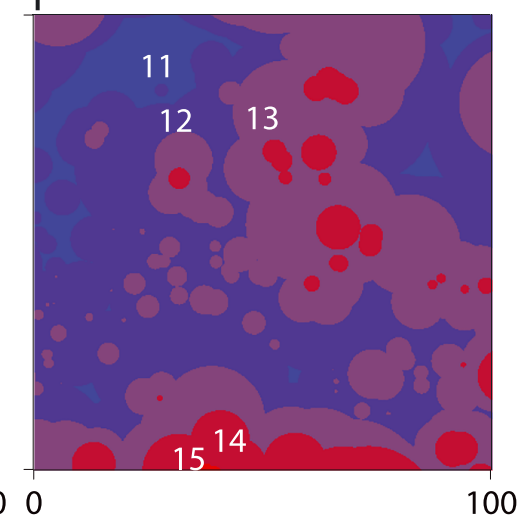

Position [km]

Figure 3. Model and convective scale increase. (a) Schematic illustrating three-cold pool (CP) collisions: for successful collision, the collision point (bold black dot) must lie within the triangle formed by the three circle centers $\left(\mathbf{c}_{1}, \mathbf{c}_{2}, \mathbf{c}_{3}\right)$ (thin black dots) shown. The red line illustrates the stationary gust front. (b) Example of CP spreading in space (different colors indicate different CPs). Panels from left to right, first and second row, indicate increasing time. Note the formation of stationary fronts when CPs interfere. Upper right panel: note the junction of three CPs without formation of a new CP (marked by a black arrow); lower panels: note the formation of several new CPs (one is highlighted by a green arrow). Further note the formation of curved fronts between these new CPs due to asynchronous timings in the second and further generations (white arrow). (c) Gust fronts simulated by the three-CP model approximately $2.5 \mathrm{hr}$ after precipitation onset when using initial cell density $N(0) A^{-1}=.01 \mathrm{~km}^{-2}$ and $c_{0}=10 \mathrm{~m} / \mathrm{s}$. Lighter gust front shades mark older, stationary, fronts. (d) Cell generations corresponding to the areas covered by the different CPs in (c). White numbers mark the CP generation corresponding to each color shading, that is, bright red corresponds to latest generation. (e, f) Similar to (c) and (d), respectively, but approximately 5 hours after precipitation onset. Note the temporally increasing spatial scale, when comparing (c) and (e) or (d) and (f), respectively.

that CP gust fronts do not travel further after collision. Where two circle perimeters eventually collide, an immobile and inactive front is therefore formed. Inactive fronts have no further effect. In each point where three circles collide, a new circle of generation two is seeded with zero initial radius. As soon as this circle is introduced, its radius also grows at speed $c_{0}$. The model hence involves a spatial growth process and a replication mechanism. Besides the restriction to three $\mathrm{CP}$ collisions, our main assumptions are that all $\mathrm{CPs}$ spread with constant and equal speed in all directions-assumptions we revisit in the conclusions.

Model implementation. The mathematical model (see results) is simulated in two ways: (1) a numerical approximate method, where a discrete domain of 2,000 $\times 2,000$ grid boxes is used, and circle centers are seeded by allocating a unique circle index to $N$ grid boxes selected randomly from a uniform distribution. Spreading in space is accomplished by sequentially copying the circle index to all neighboring grid boxes, which are within the corresponding $\mathrm{CP}$ radius. At locations where grid boxes of three different circle indexes meet and the triangle condition is fulfilled (Figure 3a), a new circle center is initialized. (2) A semianalytical method is used as an additional check of the results. A detailed description of both (1) and (2) is provided in section S1. 


\section{Results}

\subsection{Characterizing CP Interactions}

To examine how CPs interact, as a benchmark CP numerical experiment, we first analyze LES of the transient response of the convective cloud field to diurnal surface heating (simulation details: section 2.1). Positive near-surface vertical velocity $w(z=100 \mathrm{~m})$, or equivalently, low-level horizontal convergence, is a useful indicator of CP boundaries. Before the onset of precipitation, the thin boundaries of positive $w(z=100 \mathrm{~m})$ form relatively long-lived cells of typical scales $\sim 2 \mathrm{~km}$ (Figure 1a; Haerter et al., 2017). The geometry of the pattern can be characterized by a network of links (line structures) and nodes (intersects of links; Glassmeier \& Feingold, 2017). Both links and nodes, which can result from the interference of two and three CPs, respectively, correspond to updraft regions. We want to characterize the nature of the interactions and therefore investigate updraft strength for links versus nodes. In order to set off new precipitating convection, the updraft speed aloft, near the lifting condensation level ( $z \sim 1,000 \mathrm{~m})$, is relevant. High percentiles of $w(1,000 \mathrm{~m})$ serve as a proxy for locations where cloud base velocity is largest (Figures 1a-1c). Visual inspection shows that it is initially nearly always at nodes (quantified here by $w(100 \mathrm{~m})$ ), rather than along the links of low-level convergence, where $w(1,000 \mathrm{~m}$ ) is largest (Figure 1a), in qualitative agreement with theory on hexagonal Rayleigh-Bénard cells (Drazin \& Reid, 2004).

When precipitation sets in at a given location, the gust fronts initially protrude radially, forming near-circular boundaries. For a system of many CPs, the radial spreading of a given gust front is eventually reduced or halted by other fronts spreading in opposing directions-collisions result, again forming boundaries between fronts (new links). As the pattern evolves, scales increase approximately linearly under the action of CPs (Haerter et al., 2017), qualitatively seen in the coarser convergence patterns (Figures 1b and 1c). Many locations of strong $w(1,000 \mathrm{~m})$ again coincide with nodes (Böing et al., 2012). Occasionally, links and more rarely single gust front processes also cause new cells to emerge.

To quantify interaction modes, tracer particles are seeded near the edges of precipitation cells. The tracer particles are advected with the flow and thereby highlight the gust fronts belonging to each $\mathrm{CP}$ (details: section 2 and Figure S4). The histograms of $w(1,000 \mathrm{~m})$, conditional on the collocation of particles belonging to different CPs (Figure 1d), quantify the dependence of updrafts on the interaction mode. Gust fronts generally are associated with higher vertical velocities. However, where collisions between gust fronts occur, vertical velocities are larger. In the case where three gust fronts collide, velocities are even larger, with moderate dependence on the time of the model day (Figures S4d-S4f). Collisions between two CPs (2CP collisions) conceptually differ from collisions between three CPs: In 2CP collisions, air can escape both vertically and tangentially (Droegemeier \& Wilhelmson, 1985). For 3CP collisions, however, air is horizontally captured in between the three participating CPs. Only the vertical degree of freedom remains, leading to exclusively vertical motion.

\subsection{Applying the Conceptual Model}

We now turn to our conceptual model (section 3) and describe the dynamics of the diurnal cycle (Figures $1 \mathrm{a}-1 \mathrm{c})$, focusing on the three- $\mathrm{CP}(3 \mathrm{CP})$ collisions, where strong updrafts are favored. We first present an analytical mean field solution, which neglects spatial correlations, and subsequently simulate our conceptual model to obtain explicit results on spatial self-organization.

Mean Field Solution to the Conceptual Model. In a simple approximation, CPs are taken to be seeded simultaneously at discrete iteration steps $n$. Further, we assume that the cells initiated at each iteration are distributed randomly in space, that is, systematic spatial fluctuations in cell number density are ignored. Under these assumptions we estimate the mean distance, $l$, a CP needs to spread before colliding with another as the square root of the CP's mean area. With the domain length $L$ and the total number of CPs $N$, $l \approx a^{1 / 2}=L N^{-1 / 2}$, where $a \equiv A N^{-1}$ is the mean area available to any individual CP. The typical time from initiation to collision of a given $\mathrm{CP}$ will hence be

$$
\Delta t=L N^{-1 / 2}\left(2 c_{0}\right)^{-1}
$$

where the relative speed equals $2 c_{0}$, warranting the fact that the CPs approach one another.

A fraction of the collisions between three CPs is assumed to lead to a new precipitation event. It is hence meaningful to define a replication rate $r$ as the average number of new cells generated by each present cell. To give an upper bound on $r$, we use that in Voronoi graphs for random points, the average number of Voronoi 
nodes for each point is six (Yan et al., 2011); hence, six corners are expected on average for each cell. Given that three cells are involved in each collision, there can be no more than two new CPs for each previous CP, implying $r \leq 2$ (compare Figures 1 and 3a).

More explicitly, assuming that the $N(n)$ cells at iteration $n$ produce $N(n+1)=r N(n)$ new cells, then the change in the cell number from iteration $n$ to $n+1$ is

$$
\Delta N=(r-1) N(n)
$$

To obtain temporal dynamics, using equations (1) and (2), we can approximate the time derivative of $N(t)$ as

$$
\frac{\mathrm{d} N}{\mathrm{~d} t} \approx \frac{\Delta N}{\Delta t}=2 c_{0} L^{-1}(r-1) N^{3 / 2},
$$

with $\Delta t$ representing the typical time from one iteration to the next. Integrating equation (3) yields

$$
l(t) \equiv L N(t)^{-1 / 2}=l_{0}+c_{0}(1-r) t
$$

where $l(t)$ is the average distance between cell centers at time $t$ and $l_{0}$ is the integration constant. $l_{0}$ represents the typical spacing between initially seeded cells; hence, $l_{0}=L N(0)^{-1 / 2}$. For a replication rate $r>1$ scales shrink, whereas for $r<1$ cells become increasingly sparsely positioned and scales grow at a constant rate. We test this result using previous analysis of spatial scales in LES data (Haerter et al., 2017). For various different simulations, a linear increase of scale was indeed found (Figure 2a). Also, the slope of the LES simulated curves is reproduced by our analytical model (equation (4)) when choosing $r \in[0.8,0.92]$. However, $r$ remains an adjustable parameter. We therefore now aim to constrain $r$, by demanding that it emerges from the self-organization of the interacting cells.

Self-organized replication dynamics. In our mean field description, we assumed replication to occur at an adjustable rate $r$. We now allow $r$ to emerge from physically meaningful constraints on the dynamics. To this end, consider that not all interferences of three CPs are likely to trigger updrafts: when three CPs meet, depending on the relative timing and location at which the three originate, two qualitatively different geometries are possible: (i) air is enclosed by the three CPs or (ii) air escapes laterally as momentum is channeled away from the location of interference (Figure 3a). The former case can be seen as forcing air masses upward, facilitating updrafts, whereas the latter case limits the thrust of the collision. Case (i) requires the location of collision to lie within the triangle formed by the three circle centers.

To take this distinction into account, we refine our conceptual model by allowing only cases of type (i) and then simulate an initial population of thousands of cells (details: section 3), which all are seeded at time $t=0$ (example: Figure 3b). The evolving pattern of spreading circles, representing the gust fronts (Figures 3c-3f), shows a patchwork of mostly small-scale closed circular and larger-scale line structures, with overall length scales increasing over time. The circular structures at small scales are due to active $\mathrm{CP}$ gust fronts that recently emerged and have not yet collided. The larger the $\mathrm{CP}$ gust fronts become, the more likely they are to have collided with other CPs. The stationary fronts resulting from these collisions lead to generally larger-scale line structures. The patterns and the scale increase are visually comparable to the self-organizing CP gust fronts in LES (Figure 1). Also there, it is common to find small circular and larger, more line-like, structures.

Generations. Even though any cell of generation $g$ is generally produced at a different time, the positioning of the cell centers corresponding to a given generation nonetheless only allows collisions with cells of the same generation (Figures 3b, 3d, and 3f). The reason is that the cell center of any given cell of generation $g$ will be enclosed on all sides by cells of generation $g-1$, which themselves expand out from the cell center. Elements of the dynamics can best be appreciated in a one-dimensional analog (Figure S7).

In contrast to the simplified mean field description, cells belonging to any given iteration will not emerge simultaneously. However, we use that only cells of equal generations can collide (Figure $3 \mathrm{~b}$ ). We thus assign a label of generation $g=1$ to initial cells and record all subsequent generations: any successful collision between generation $g$ cells yields a generation $g+1$ cell. Collecting all cells of any generation $g$ and computing the average time $t(g)$ when these cells are created, we find that $N(t(g))$ does indeed approximately follow the functional form suggested by equation (4) (Figure 2b). However, the large $t$ asymptotic behavior shows 
a statistically significant departure from the exponent -2 implied by equation (4). This deviation requires that the assumption in equation 1 should be generalized as

$$
\Delta t=L N^{-\alpha}\left(2 c_{0}\right)^{-1},
$$

thus yielding a more general version of equation (4)

$$
N^{-\alpha}=\frac{2 \alpha(1-r)}{L} c_{0} t+\text { const }
$$

In equations (5) and (6), the exponent $\alpha \neq 1 / 2$ takes into account that there may be a systematically nonrandom organization of cells in space. In the limit of large $t$, we have $\lim _{t \rightarrow \infty} N(t) \sim t^{-1 / \alpha}$, hence, to fit the dependency $\lim _{t \rightarrow \infty} N(t) \sim t^{-1.7}$ (Figure $2 \mathrm{~b}$ ), $\alpha \approx 0.6$ is required, indicating a measurable departure from a random configuration.

Cell clustering. The deviation from $\alpha=1 / 2$ suggests that cells might be clustered. To explore this, we analyze the spatial pattern formed by cell centers initiated during a finite time window (Figures $2 \mathrm{~d}$ and $2 \mathrm{~b}$, inset). We break the domain down into 400 subregions of equal 100-km $\times 100-\mathrm{km}$ areas, and within each subregion enumerate the number of cell centers during a 3-hr time interval. The probability distribution function of all counts in the subregions is compared to a shuffled counterpart, where cell centers are distributed at random over the domain during the same time interval (details: see section S3). The analysis shows that the likelihood of finding extreme concentrations of cells in a subregion increases for the self-organized results relative to random spatial distributions of cells-a feature that can also be noted qualitatively when inspecting the patchiness of the spatial pattern (Figure 2b, inset). Repeating for a smaller subregion (Figure S8), on the order of a metropolitan area, we show that clustering occurs also there.

We further point out the nonconstant replication rate $r(g) \equiv N(g+1) / N(g)$ (Figure 2c). For an initially random spatial configuration and synchronous cell initiation, we numerically verify that $r(1)=1$. The value of $r(g)$ subsequently degrades and eventually saturates near $r \approx 0.87$ for $n>20$. Lack of synchrony for $g>1$ indeed is a plausible explanation for this gradual reduction of $r$. It is easy to show that two simultaneously seeded cells can "swallow" a cell seeded at a later time; that is, the Voronoi cells corresponding to the earlier points can entirely enclose the Voronoi cell corresponding to the later one (Kim et al., 2001). Such enclosures would reduce the effective number of nodes each point can produce, hence causing $r<1$.

Our model assumes that each 3CP collision yields a new deep convective event, but collisions will likely occasionally fail at doing so, hence $r$ might be somewhat lower than the value obtained here. To account for such imperfect replication, appropriately reduced $c_{0}$ should be considered. In reality, however, there may also be other sources of new cells than $3 \mathrm{CP}$ collisions.

\section{Discussion}

Previous work suggested that stronger CPs could enhance precipitation extremes (Haerter \& Schlemmer, 2018). Describing extremes may be accomplished by our model, by coupling precipitation intensity and gust front speed, thereby relaxing the assumption of constant speed $c_{0}$. The actual speed of CP gust fronts, even in a crude approximation, will depend on the initial virtual potential temperature perturbation $\Delta \Theta$ caused by precipitation evaporation, sometimes approximated as $c(\Delta \Theta, h)=\sqrt{2 g h(\Delta \Theta / \Theta)}$ (Etling, 2008). Here, $h$ denotes the effective $\mathrm{CP}$ height, and $g$ is the gravitational acceleration. We speculate that, when gust fronts of larger speed collide, more environmental air might be enclosed in the resulting updraft. Further, due to the effect of surface heat fluxes and energy conservation, the speed of spreading should decrease with radius (Grant \& van den Heever, 2016, 2018; Gentine et al., 2016; Romps \& Jeevanjee, 2016). The exact dependence on radius hinges on factors such as drag and mixing, which is a matter of current debate. Extensions of our model that incorporate precipitation intensity and its effect on CP momentum could be used to guide the analysis of extreme precipitation events and how they relate to CP collisions.

Furthermore, our model implies the existence of generations of cells and the lack of interaction between cells of distinct generations. It should be considered whether observations, such as those from satellite data, fully support this claim. When the gust front of one generation is fast enough to catch up with that of a previous generation, our postulate may be violated. In its defense, one should then however also consider the appreciable time delay between an updraft, leading to precipitation and CP formation. Such time delays would diminish the ability of a subsequent gust front catching up with a previous one. 
Acknowledgments

We thank Guillermo García-Peréz, Christopher Moseley, Kim Sneppen, and Peter Berg for fruitful discussions on the mathematical model. We are grateful to both anonymous reviewers for their in-depth comments to our manuscript. O. H. and J. O. H. gratefully acknowledge funding by a grant (13168) from the VILLUM Foundation. This project has received funding from the European Research Council (ERC) under the European Union's Horizon 2020 research and innovation program (grant agreement 771859). S. J. B. is partially funded through the NERC/Met Office Joint Programme on Understanding and Representing Atmospheric Convection across Scales (GENESIS, grant NE/N013840/1). S. B. N. acknowledges funding through the Danish National Research Foundation (grant DNRF116). The simulation data and program code used for the LES analysis are available from Moseley et al. (2016). The authors are grateful for computing resources and technical assistance provided by the Danish Center for Climate Computing, a facility built with support of the Danish e-Infrastructure Corporation, Danish Hydrocarbon Research and Technology Centre, VILLUM Foundation, and the Niels Bohr Institute.
In reality, CPs cannot spread indefinitely. Observed maximal radii in fact vary by an order of magnitude, between 10 and 100 km (Black, 1978; Feng et al., 2015; Zuidema et al., 2012). For larger-scale phenomena, such as the near-planetary organization observed in the Madden-Julian Oscillation (Zhang, 2005), it may be crucial to consider the constraints on maximum CP radius. This may also help to better assess models of self-organization, such as those employed in the idealized self-aggregation case (Holloway, 2017; Holloway et al., 2017; Wing \& Cronin, 2016; Wing et al., 2017; Yang, 2018). The detailed effects of CPs on self-aggregation are currently not conclusively understood, but CP strength has been mentioned as modifying the ability of simulations to show self-aggregation (Jeevanjee \& Romps, 2013; Muller \& Bony, 2015)

Our results on clustering (Figure 2d) may have implications for extreme events, such as flash floods. The scales chosen in our example reflect realistic spatial $(\sim 10-100 \mathrm{~km})$ and temporal $(\sim 1-3 \mathrm{hr})$ accumulation intervals (Golding et al., 2016; Jones et al., 2014; Kendon et al., 2014; Olsson et al., 2017), and our analysis suggests that self-organization boosts the likelihood of flash floods compared to random spatial organization. While qualitative, this finding should be explored in more detail to decipher, which impact clustering can have on the prediction of extremes, today and in a climate with modified near-surface temperature.

Modifications to our model could further allow for refractory effects of CPs: the likelihood of updrafts forming in regions previously affected by CPs is reduced due to negative buoyancy resulting from the evaporative cooling. Such areas would effectively function as traps, where collisions would not have any pronounced effect in generating updrafts. Our analysis has further assumed zero-wind shear conditions. Wind shear is however known to induce organization along quasi-linear geometries, such as in the case of squall line convection (Rotunno et al., 1988). Variations of our model, which allow for anisotropic spreading of CPs, mimicking the effect of wind shear in squall lines, along with an implementation of negative buoyancy, could be sufficient to allow for the formation of more linear structures as found in squall lines.

As new cells are often generated when three CPs meet, we here developed a conceptual model based on 3CP collisions only. Alternatively, one could imagine conceptual models based on $2 \mathrm{CP}$ collisions or combinations of 2 and $3 \mathrm{CP}$ collisions. Such models could also lead to scale changes with time. However, additional considerations, such as the probability of a collision in fact leading to the formation of a new cell center, would have to be taken into account. Ultimately, generalizations of the model described here could kick-start new attempts at parameterizing convective organization in large-scale models.

\section{Conclusion}

CP interactions are observed ubiquitously over land (Engerer et al., 2008) and sea (de Szoeke et al., 2017; Feng et al., 2015), and LES allow for the analysis of their dynamics (Haerter \& Schlemmer, 2018; Moseley et al., 2016; Schlemmer \& Hohenegger, 2015; Tompkins, 2001; Torri et al., 2015). LES incorporate many of the physical processes that are relevant in describing the formation and dynamics of CPs: three-dimensional fluid dynamics with appropriate boundary conditions, condensation and evaporation effects, precipitation formation, and radiative transfer. Using LES, we here pinpoint in which ways CPs interact during the diurnal cycle and that the collision of gust fronts from three CPs is crucial for cloud formation. We then formulate a simple conceptual model where we represent CPs as circles that grow with equal and constant radial speed. The model is initialized with circles that expand from randomly located points in 2-D space. Replication of CPs is introduced by seeding a new expanding circle from the point where three circles collide. We show that this model allows us to reproduce the dynamics of CP gust fronts found in the LES. Our model, intentionally left simple, captures the essential aspects of diurnal cycle dynamics, where spatial scales increase linearly with time. The model highlights the complexity of convective self-organization through CPs and offers a framework for the description of clustering and extremes.

\section{References}

Bengtsson, L., Steinheimer, M., Bechtold, P., \& Geleyn, J.-F. (2013). A stochastic parametrization for deep convection using cellular automata. Quarterly Journal of the Royal Meteorological Society, 139(675), 1533-1543.

Berg, P., Moseley, C., \& Haerter, J. O. (2013). Strong increase in convective precipitation in response to higher temperatures. Nature Geoscience, 6(3), 181-185.

Black, P. G. (1978). Mesoscale cloud patterns revealed by apollo-soyuz photographs. Bulletin of the American Meteorological Society, 59(11), 1409-1419. 
Böing, S. J. (2016). An object-based model for convective cold pool dynamics. Mathematics of Climate and Weather Forecasting, 2(1), 43-60. Böing, S. J., Jonker, H. J., Siebesma, A. P., \& Grabowski, W. W. (2012). Influence of the subcloud layer on the development of a deep convective ensemble. Journal of the Atmospheric Sciences, 69(9), 2682-2698.

Cafaro, C., \& Rooney, G. G. (2018). Characteristics of colliding density currents: A numerical and theoretical study. Quarterly Journal of the Royal Meteorological Society, 144, 1761-1771.

de Szoeke, S. P., Skyllingstad, E. D., Zuidema, P., \& Chandra, A. S. (2017). Cold pools and their influence on the tropical marine boundary layer. Journal of the Atmospheric Sciences, 74(4), 1149-1168.

Drager, A. J., \& van den Heever, S. C. (2017). Characterizing convective cold pools. Journal of Advances in Modeling Earth Systems, 9, 1091-1115. https://doi.org/10.1002/2016MS000788

Drazin, P. G., \& Reid, W. H. (2004). Hydrodynamic stability (p. 58). Cambridge, UK: Cambridge university press.

Droegemeier, K. K., \& Wilhelmson, R. B. (1985). Three-dimensional numerical modeling of convection produced by interacting thunderstorm outflows. Part I: Control simulation and low-level moisture variations. Journal of the Atmospheric Sciences, 42(22), $2381-2403$.

Engerer, N. A., Stensrud, D. J., \& Coniglio, M. C. (2008). Surface characteristics of observed cold pools. Monthly Weather Review, 136(12), 4839-4849.

Etling, D. (2008). Theoretische Meteorologie: Eine Einführung. Berlin: Springer-Verlag.

Feingold, G., Koren, I., Wang, H., Xue, H., \& Brewer, W. A. (2010). Precipitation-generated oscillations in open cellular cloud fields. Nature, 466(7308), 849-852. https://doi.org/10.1038/nature09314

Feng, Z., Hagos, S., Rowe, A. K., Burleyson, C. D., Martini, M. N., \& Szoeke, S. P. (2015). Mechanisms of convective cloud organization by cold pools over tropical warm ocean during the AMIE/DYNAMO field campaign. Journal of Advances in Modeling Earth Systems, 7 , 357-381. https://doi.org/10.1002/2014MS000384

Gentine, P., Garelli, A., Park, S.-B., Ji, N., Torri, G., \& Kuang, Z. (2016). Role of surface heat fluxes underneath cold pools. Geophysical research letters, 43, 874-883. https://doi.org/10.1002/2015GL067262

Glassmeier, F., \& Feingold, G. (2017). Network approach to patterns in stratocumulus clouds. Proceedings of the National Academy of Sciences, 114, 10,578-10,583.

Golding, B., Roberts, N., Leoncini, G., Mylne, K., \& Swinbank, R. (2016). Mogreps-uk convection-permitting ensemble products for surface water flood forecasting: Rationale and first results. Journal of Hydrometeorology, 17(5), 1383-1406.

Grandpeix, J.-Y., \& Lafore, J.-P. (2010). A density current parameterization coupled with Emanuel's convection scheme. Part I: The models. Journal of the Atmospheric Sciences, 67(4), 881-897.

Grandpeix, J.-Y., Lafore, J.-P., \& Cheruy, F. (2010). A density current parameterization coupled with Emanuel's convection scheme. Part II: 1D simulations. Journal of the Atmospheric Sciences, 67(4), 898-922.

Grant, L. D., \& van den Heever, S. C. (2016). Cold pool dissipation. Journal of Geophysical Research: Atmospheres, 121, 1138-1155. https:// doi.org/10.1002/2015JD023813

Grant, L. D., \& van den Heever, S. C. (2018). Cold pool-land surface interactions in a dry continental environment. Journal of Advances in Modeling Earth Systems, 10(7), 1513-1526. https://doi.org/10.1029/2018MS001323

Guerreiro, S. B., Fowler, H. J., Barbero, R., Westra, S., Lenderink, G., Blenkinsop, S., et al. (2018). Detection of continental-scale intensification of hourly rainfall extremes. Nature Climate Change, 8, 803-807.

Haerter, J. O., Berg, P., \& Moseley, C. (2017). Precipitation onset as the temporal reference in convective self-organization. Geophysical Research Letters, 44, 6450-6459. https://doi.org/10.1002/2017GL073342

Haerter, J. O., \& Schlemmer, L. (2018). Intensified cold pool dynamics under stronger surface heating. Geophysical Research Letters, 45, 6299-6310. https://doi.org/10.1029/2017GL076874

Holloway, C. E. (2017). Convective aggregation in realistic convective-scale simulations. Journal of Advances in Modeling Earth Systems, 9 , 1450-1472. https://doi.org/10.1002/2017MS000980

Holloway, C. E., Wing, A. A., Bony, S., Muller, C., Masunaga, H., L'Ecuyer, T. S., et al. (2017). Observing convective aggregation. Surveys in Geophysics, 38(6), 1199-1236.

Jeevanjee, N., \& Romps, D. M. (2013). Convective self-aggregation, cold pools, and domain size. Geophysical Research Letters, 40, 994-998. https://doi.org/10.1002/grl.50204

Jeevanjee, N., \& Romps, D. M. (2015). Effective buoyancy, inertial pressure, and the mechanical generation of boundary layer mass flux by cold pools. Journal of the Atmospheric Sciences, 72(8), 3199-3213.

Jones, M. R., Blenkinsop, S., Fowler, H. J., \& Kilsby, C. G. (2014). Objective classification of extreme rainfall regions for the uk and updated estimates of trends in regional extreme rainfall. International Journal of Climatology, 34(3), 751-765.

Kendon, E. J., Roberts, N. M., Fowler, H. J., Roberts, M. J., Chan, S. C., \& Senior, C. A. (2014). Heavier summer downpours with climate change revealed by weather forecast resolution model. Nature Climate Change, 4, 570-576.

Khairoutdinov, M., \& Randall, D. (2006). High-resolution simulation of shallow-to-deep convection transition over land. Journal of the Atmospheric Sciences, 63(12), 3421-3436.

Kim, D.-S., Kim, D., \& Sugihara, K. (2001). Voronoi diagram of a circle set from Voronoi diagram of a point set: II. geometry. Computer Aided Geometric Design, 18(6), 563-585.

Koren, I., \& Feingold, G. (2011). Aerosol-cloud-precipitation system as a predator-prey problem. Proceedings of the National Academy of Sciences of the United States of America, 108(30), 12227-32. https://doi.org/10.1073/pnas.1101777108

Lenderink, G., \& van Meijgaard, E. (2008). Increase in hourly precipitation extremes beyond expectations from temperature changes. Nature Geoscience, 1, 511-514.

Lima, M. A., \& Wilson, J. W. (2008). Convective storm initiation in a moist tropical environment. Monthly Weather Review, 136(6), $1847-1864$.

Mellado, J. P. (2012). Direct numerical simulation of free convection over a heated plate. Journal of Fluid Mechanics, 712, 418-450.

Moncrieff, M. W. (2010). The multiscale organization of moist convection and the intersection of weather and climate. Climate Dynamics: Why Does Climate Vary? 189, 3-26.

Moseley, C., Berg, P., \& Haerter, J. O. (2013). Probing the convection life-cycle by iterative rain cell tracking. Journal of Geophysical Research: Atmospheres, 118, 13,361-13,370. https://doi.org/10.1002/2013JD020868

Moseley, C., Henneberg, O., \& Haerter, J. O. (2019). A statistical model for isolated convective precipitation events. Journal of Advances in Modeling Earth Systems, 11, 360-375. https://doi.org/10.1029/2018MS001383

Moseley, C., Hohenegger, C., Berg, P., \& Haerter, J. (2016). Convective extremes driven by cloud-cloud interaction. Nature Geoscience, 9 , 748-752.

Muller, C., \& Bony, S. (2015). What favors convective aggregation and why? Geophysical Research Letters, 42, 5626-5634. https://doi.org/ 10.1002/2015GL064260 
Olsson, J., Pers, B. C., Bengtsson, L., Pechlivanidis, I., Berg, P., \& Körnich, H. (2017). Distance-dependent depth-duration analysis in high-resolution hydro-meteorological ensemble forecasting: A case study in malmö city, sweden. Environmental Modelling \& Software, 93, 381-397.

Pincus, R., \& Stevens, B. (2009). Monte Carlo spectral: A consistent approximation for radiative transfer in large eddy simulations. Journal of Advances in Modeling Earth Systems, 1, 1. https://doi.org/10.3894/JAMGES.2009.1.1

Purdom, J. (1982). Subjective interpretation of geostationary satellite data for nowcasting. Nowcasting, 149-166.

Rio, C., Hourdin, F., Grandpeix, J.-Y., \& Lafore, J.-P. (2009). Shifting the diurnal cycle of parameterized deep convection over land. Geophysical Research Letters, 36, L07809. https://doi.org/10.1029/2008GL036779

Romps, D. M., \& Jeevanjee, N. (2016). On the sizes and lifetimes of cold pools. Quarterly Journal of the Royal Meteorological Society, 142(696), 1517-1527.

Rotunno, R., Klemp, J. B., \& Weisman, M. L. (1988). A theory for strong, long-lived squall lines. Journal of the Atmospheric Sciences, 45(3), 463-485.

Schlemmer, L., \& Hohenegger, C. (2015). Modifications of the atmospheric moisture field as a result of cold-pool dynamics. Quarterly Journal of the Royal Meteorological Society, 142, 30-42. https://doi.org/10.1002/qj.2625

Seifert, A., \& Beheng, K. (2006). A two-moment cloud microphysics parameterization for mixed-phase clouds. Part 1: Model description. Meteorology and Atmospheric Physics, 92(1-2), 45-66.

Stevens, B., Moeng, C.-H., Ackerman, A. S., Bretherton, C. S., Chlond, A., de Roode, S., et al. (2005). Evaluation of large-eddy simulations via observations of nocturnal marine stratocumulus. Monthly Weather Review, 133(6), 1443-1462.

Tompkins, A. M. (2001). Organization of tropical convection in low vertical wind shears: The role of cold pools. Journal of the Atmospheric Sciences, 58(13), 1650-1672.

Torri, G., \& Kuang, Z. (2016). Rain evaporation and moist patches in tropical boundary layers. Geophysical Research Letters, 43, 9895-9902. https://doi.org/10.1002/2016GL070893

Torri, G., Kuang, Z., \& Tian, Y. (2015). Mechanisms for convection triggering by cold pools. Geophysical Research Letters, 42, 1943-1950. https://doi.org/10.1002/2015GL063227

Wing, A. A., \& Cronin, T. W. (2016). Self-aggregation of convection in long channel geometry. Quarterly Journal of the Royal Meteorological Society, 142(694), 1-15.

Wing, A. A., Emanuel, K., Holloway, C. E., \& Muller, C. (2017). Convective self-aggregation in numerical simulations: A review. Surveys in Geophysics, 38(6), 1173-1197.

Yan, D.-M., Wang, K., Lévy, B., \& Alonso, L. (2011). Computing 2D periodic centroidal Voronoi tessellation. In Voronoi Diagrams in Science and Engineering (ISVD), 2011 Eighth International Symposium on (pp. 177-184). IEEE.

Yang, D. (2018). Boundary layer diabatic processes, the virtual effect, and convective self-aggregation. Journal of Advances in Modeling Earth Systems, 10, 2163-2176. https://doi.org/10.1029/2017MS001261

Zhang, C. (2005). Madden-Julian Oscillation. Reviews of Geophysics, 43, RG2003. https://doi.org/10.1029/2004RG000158

Zuidema, P., Li, Z., Hill, R. J., Bariteau, L., Rilling, B., Fairall, C., et al. (2012). On trade wind cumulus cold pools. Journal of the Atmospheric Sciences, 69(1), 258-280.

Zuidema, P., Torri, G., Muller, C., \& Chandra, A. (2017). A survey of precipitation-induced atmospheric cold pools over oceans and their interactions with the larger-scale environment. Surveys in Geophysics, 38, 1283-1305. 